The University of Kansas

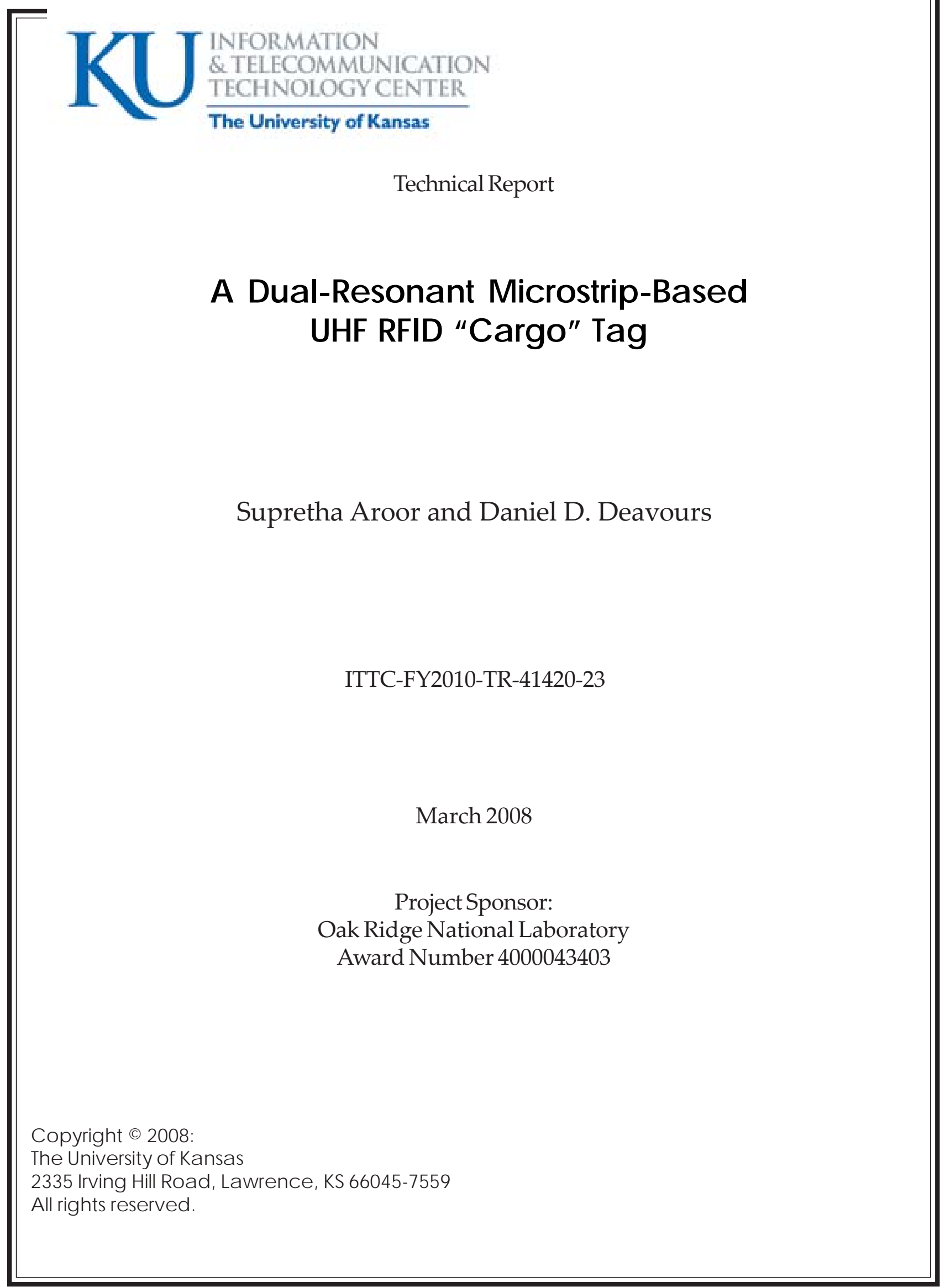




\title{
A Dual-Resonant Microstrip-Based UHF RFID “Cargo” Tag
}

\author{
Supreetha Aroor and Daniel D. DeavoursInformation and Telecommunications Technology \\ Center \\ University of Kansas, Lawrence, KS \\ Email: \{saroor,deavours\}@ittc.ku.edu
}

\begin{abstract}
We present a novel passive UHF RFID "cargo tag" capable of operating in two different graphic regions such as Europe and North America. The tag utilizes a dual-resonant, dual-polarized, microstrip antenna. The resulting tag operates efficiently over ETSI and FCC frequency ranges and achieves excellent efficiency. The tag antenna eliminates any cross-layer structures such as a via and may be manufactured efficiently using traditional, low-cost "inlay" technology. We estimate the free-space read distance to be between 9.3 and 13.1 meters (30 and 43 feet).
\end{abstract}

\section{Index Terms}

Antennas, RFID, Antenna feeds, Microstrip antennas, Multifrequency antennas, Impedance matching.

This work was supported by the Information and Telecommunications Technology Center at the University of Kansas, the Office of Naval Research through Award Number N00014-07-1-1042, Oak Ridge National Laboratory via Award Number 4000043403, and the KU Transportation Research Institute. 
CONTENTS

I Introduction $\quad 3$

II Background and Related Work 3

III Antenna Design $\quad 5$

$\begin{array}{llr}\text { IV Measurements } & 7\end{array}$

$\begin{array}{llr}\text { V Conclusion } & 9\end{array}$

$\begin{array}{lr}\text { References } & 10\end{array}$

LIST OF FIGURES

1 Dual-resonant, dual-polarized planar microstrip-based RFID tag. . . . . . . . . . . . . . 6

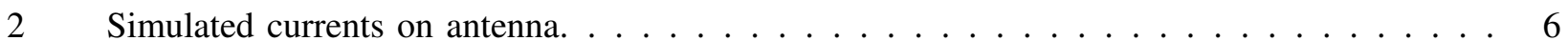

3 Power wave Smith chart of measured antenna impedance (normalized to $13-j 65 \Omega$ ). The $90 \%$ and $50 \%$ power transfer efficiency circles are also plotted. . . . . . . . . . 7

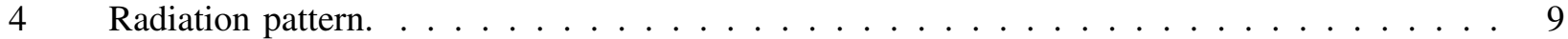




\section{INTRODUCTION}

Inexpensive, passive UHF RFID antennas are typically constructed using stripline dipole antennas [1]. The antennas are often electrically short in order to fit on a 4-inch label and narrow in order to minimize cost. Dipole antennas experience degraded performance when they are placed on or close to metal objects [2], [3], [4], and thus are not well suited for tracking large metal assets.

One of the practical advantages of modern, efficient UHF RFID ICs is the ability to identify tags at long distances. This is particularly useful for identifying metal containers, such as cargo containers. Such assets are ideal applications of UHF RFID for many reasons: they tend to have a long useful life, allowing the tag cost to be amortized over many uses; the contents change frequently, so associations with a unique identifier is useful; they frequently cross organizational boundaries, making a common mode of identification useful; and they are physically large, requiring identification at distance. Further, cargo containers are commonly shipped between large geographic regions, and different regions have different frequencies allocated for RFID. Worldwide, the UHF RFID frequency spectrum ranges roughly between 860-960 MHz range. Practical considerations require a low profile and moderate form factor, and cost is often a primary driver in such systems.

In this paper, we present a nearly four-inch square microstrip antenna placed over a six-inch square dielectric and ground plane adopted for low-cost RFID tagging of large, metal objects such as cargo containers. The antenna is dual-resonant so as to operate in two of the three frequency bands used for UHF RFID worldwide. Finally, due to a large antenna gain from the microstrip antenna, e.g., 5 dB larger than a dipole, we were able to validate the tag performance informally by reading the tag over 60 feet (18.3 meters) away in an outdoor environment using a commodity RFID reader (likely utilizing a ground reflection for $3 \mathrm{~dB}$ performance improvement). We estimate the free-space read distance to be between 9.3 and 13.1 meters.

\section{BACKGROUND AND RELATED WORK}

Microstrip "patch" antennas are a well-known class of antennas (e.g., [5]). A microstrip antenna consists of: the primary radiating element ("antenna"), a dielectric substrate, a ground plane, and a feeding element. Common feeds include a probe feed from a coaxial cable or edge feed with a microstrip transmission line, although proximity feeds and coupling through a slot in the ground plane are also possible.

Traditional approaches to microstrip-based RFID tags take these familiar structures and adapt them to the particularities of RFID. Unfortunately, the most common feed structures are based on some sort of unbalanced transmission line, whether it be a coaxial cable with a probe feed, or a microstrip transmission 
line with an edge feed. The need to establish an electrical reference ("ground") complicates the structure, usually requiring a via to the ground plane (e.g., [6]).

The via significantly complicates the manufacturing process. The UHF RFID industry commonly uses high-speed, web-based processing to manufacture "inlays." An inlay is a printed or etched antenna on a flexible substrate with a RFID chip attached, which can be manufactured with a web-based process in high volumes and at low cost. Another challenge with traditional microstrip antennas is a narrow bandwidth and single resonant frequency. Unfortunately, different regions utilize different frequency ranges within the UHF spectrum for RFID use. For example, North America uses 902-928 MHz, Europe has allocated 865-868 MHz, and Japan allocated 952-954 MHz. Most other countries of the world utilize some subset of $865-868$ or $902-928 \mathrm{MHz}$. It becomes a significant challenge to make a tag operate well over the entire 902-928 MHz frequency range and retain a low profile, small form factor, and high performance. Operating over two bands, e.g., cargo containers shipping supplies between North America and Europe, becomes especially challenging.

By feeding a rectangular microstrip antenna along the diagonal (with a probe feed), one can excite both the $T M_{01}$ and $T M_{10}$ modes [7], where the two modes have orthogonal polarization, if the two modes are sufficiently separated. The antenna dimensions can be shortened and the resonant frequencies controlled by cutting a cross-shaped slot into the antenna [8].

Commonly, antennas are designed to match to a substantially real load, e.g., a 50 or 75 Ohm transmission line, in order to minimize standing waves along the transmission line. With RFID antennas, the load is both in close proximity to the antenna and substantially reactive. Thus, the objective to optimize RFID tag performance is not to minimize the voltage reflection coefficient, but rather to maximize the power transferred to the IC (load). The remainder of this section follows from [9], [10].

Let $Z_{a}$ and $Z_{c}$ be the antenna and chip impedance respectively. Maximum power transfer is achieved when $Z_{a}=Z_{c}^{*}$, in which half the power is transferred to the load and half is scattered. The power transfer efficiency is the ratio of the actual power transferred to the maximum possible power transferred (a conjugate match), which is given by

$$
\tau=\frac{4 R_{a} R_{c}}{\left|Z_{a}+Z_{c}\right|^{2}}
$$

Similarly, a power wave reflection can be defined as

$$
s=\frac{Z_{c}-Z_{a}^{*}}{Z_{a}+Z_{c}}
$$

so that $\tau+|s|^{2}=1$. Here, $s$ plays a similar role to $S_{11}$ in the traditional antenna matching problem. 
Let

$$
\hat{z}_{a}=r+j x=\frac{R_{a}}{R_{c}}+j \frac{X_{a}+X_{c}}{R_{c}} .
$$

It can be shown that $s=\frac{\hat{z}_{a}-1}{\hat{z}_{a}+1}$, i.e., $s$ is a Smith chart transformation. The antenna (source) impedance can be plotted on a Smith chart and the distance from $\hat{z}_{a}$ to the center of the Smith chart is $|s|$. We call the Smith chart normalized in this way a power wave Smith chart.

\section{Antenna Design}

The inspiration for the antenna design comes from a combination of three factors. First, we draw from the dual-resonant rectangular antennas closely exciting the $T M_{01}$ and $T M_{10}$ modes and feeding in such a way to excite both modes [7]. Second, we draw from the use of microstrip transmission lines to build a completely planar microstrip antenna [11]. Third, we note the use of a cross-shaped slot in a microstrip antenna originally used to reduce the size of the antenna [8], but instead we use the cross as space to place our matching circuit and IC. The slots can also be used to resize the antenna as necessary to control the form factor.

Fig. 1 shows the antenna geometry, where the darkened area represents the metalized area. The antenna was designed to operate over a polypropylene substrate $5.08 \mathrm{~mm}$ thick and a dielectric constant of 2.28 and loss tangent estimated to be 0.001 . The substrate and ground plane are 6 inches $(15.2 \mathrm{~cm})$ square. The initial design to use a simple cross resulted in a matching circuit with a reactance that was too large for our IC. To reduce the reactance, we shortened the transmission lines in the matching circuit by imposing a diamond-shape cut-out within the cross.

The cross segments have a length of $34 \mathrm{~mm}$ and width of $8 \mathrm{~mm}$. The diamond is approximately $19.8 \mathrm{~mm}$ per side. The center of the feed lines are $15 \mathrm{~mm}$ from the center and $2 \mathrm{~mm}$ wide in order to achieve the desired input resistance, and the length of the feeds can be adjusted to achieve a reactance of approximately $j 65 \Omega$ at resonance. The matching circuit was designed to obtain a perfect conjugate match to $13-j 65 \Omega$ at $867 \mathrm{MHz}$, and a slightly larger real resistance at $915 \mathrm{MHz}$ to to achieve larger bandwidth.

When the first resonant mode is excited, one of the feeds acts as an inset microstrip feed, while the other feed lies primarily along the axis of symmetry and thus acts as a feed from an electrical reference, i.e., the virtual ground. When the second mode is excited, the role of the two feeds reverse. Fig. 2 illustrate the simulated currents on the antenna at the two resonant frequencies. One can see that the $T M_{01}$ and $T M_{10}$ modes are clearly excited with only a minor diagonal component excited at each frequency. 


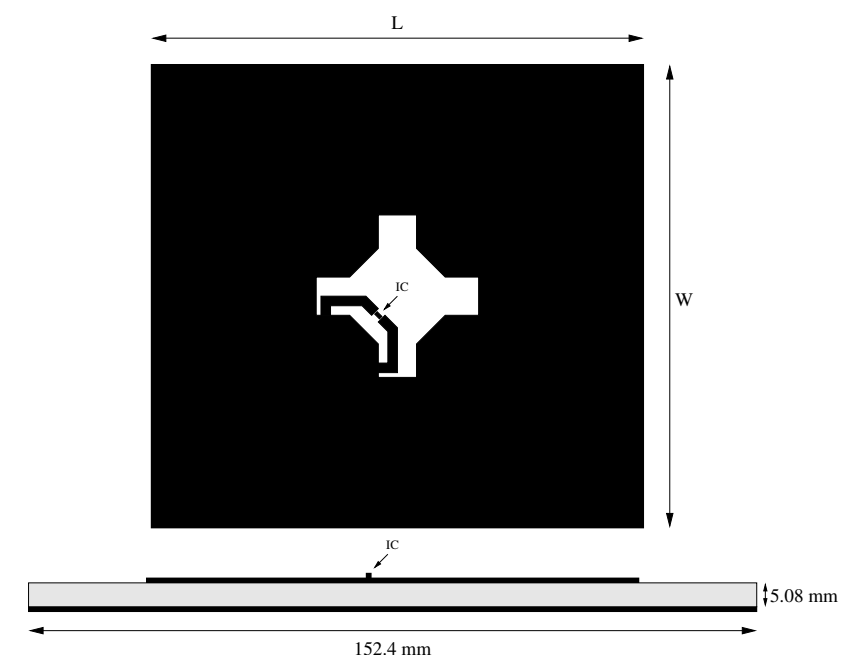

Fig. 1. Dual-resonant, dual-polarized planar microstrip-based RFID tag.

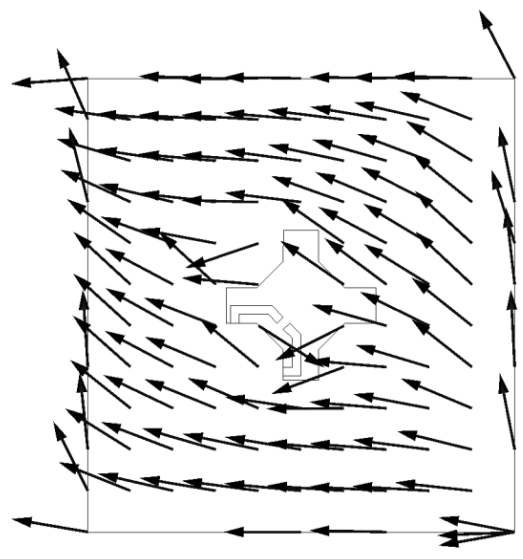

(a) $870 \mathrm{MHz}$.

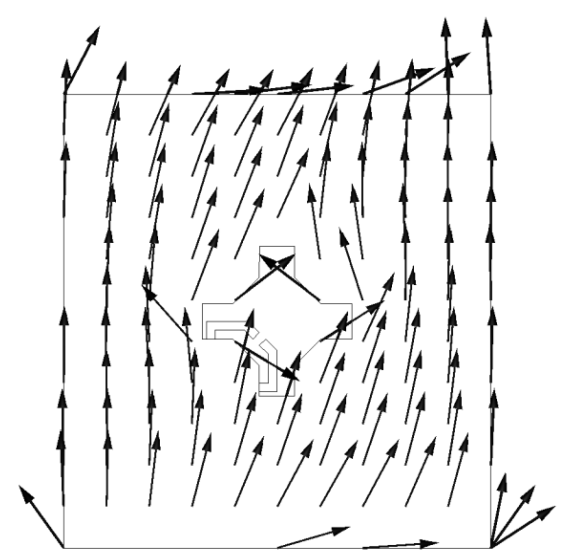

(b) $915 \mathrm{MHz}$.

Fig. 2. Simulated currents on antenna.

The two resonant frequencies can be easily adjusted by adjusting $L$ and $W$. Here, we present resonant frequencies of 867 and $915 \mathrm{MHz}$ using $L=103 \mathrm{~mm}$ and $W=97 \mathrm{~mm}$. To achieve resonant frequencies of 915 and $953 \mathrm{MHz}$, set $L=97 \mathrm{~mm}$ and $W=92.5 \mathrm{~mm}$. For resonant frequencies at $867 \mathrm{and} 953 \mathrm{MHz}$, set $L=103 \mathrm{~mm}$ and $W=92.5 \mathrm{~mm}$. Matching to a larger or smaller resistance is achieved by placing the feed lines further apart or closer together, respectively. If the impedance is too small, the size of the cross may need to be expanded to facilitate a larger $R_{a}$. A smaller $X_{a}$ can be achieved by shortening the feed lines, and a larger $X_{a}$ by longer feed lines. In this way, the antenna can be adjusted to work 


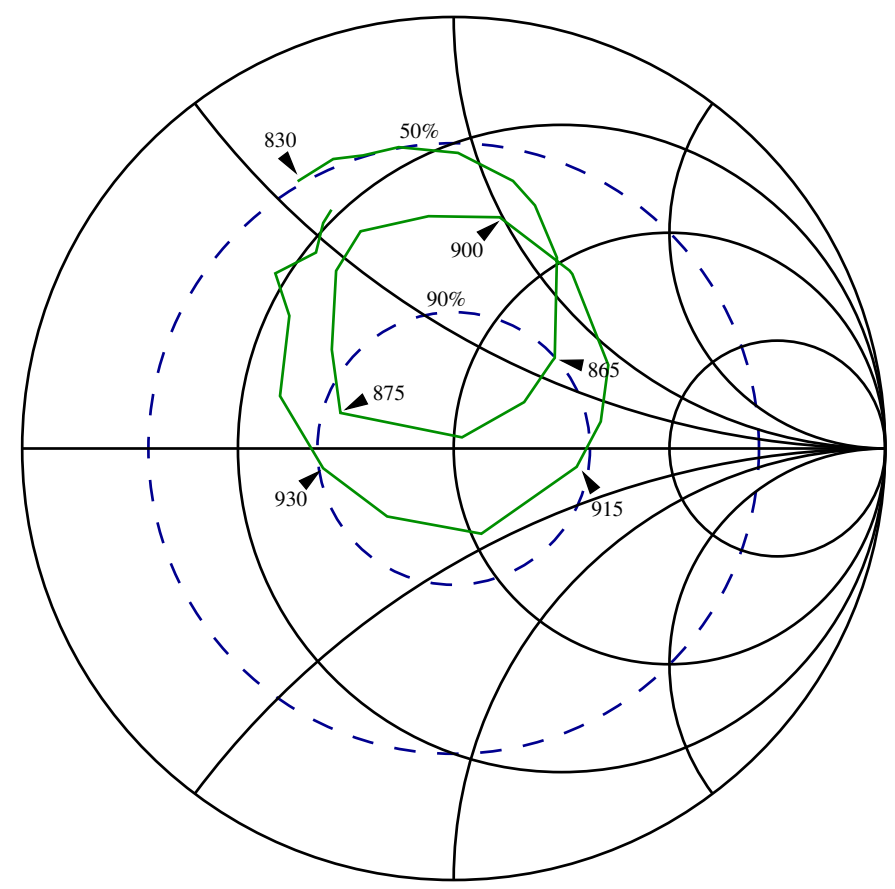

Fig. 3. Power wave Smith chart of measured antenna impedance (normalized to $13-j 65 \Omega$ ). The $90 \%$ and $50 \%$ power transfer efficiency circles are also plotted.

at any two of the three major frequencies of operation worldwide and with most commercially-available RFID ICs.

\section{MeAsurements}

As the antenna impedance is considerably reactive and balanced, it is difficult to use standard microwave measurement techniques. We constructed a measurement apparatus using a chip balun to measure the impedance using a network analyzer. The balun is mounted on a small PCB that converts a SMA signal to a differential signal to short pins, which are used to probe the IC connection pads. The apparatus yields only modest accuracy, but sufficient for our purposes here. The impedance measurements are taken over the range of 830 to $960 \mathrm{MHz}$. Note also that the impedance is near the edge of a $50 \mathrm{Ohm}$ Smith chart, and thus the accuracy of the network analyzer is likely to be relatively poor. The power wave Smith chart (cf. Section II) using the normalized impedance $13-j 65 \Omega$ is plotted in Fig. 3.

Note that we are able to achieve an almost perfect conjugate match at $870 \mathrm{MHz}$. the $90 \%$ transfer efficiency spans between 864 and $875 \mathrm{MHz}$, and between 915 and $930 \mathrm{MHz}$. At $900 \mathrm{MHz}, \tau=70 \%$. By making the antenna slightly less inductive, the $1 \mathrm{~dB}$ bandwidth would span between 860 and 940 
$\mathrm{MHz}$, and the $3 \mathrm{~dB}$ bandwidth would cover from less than 830 to more than $960 \mathrm{MHz}$.

The simulated results of the antenna using a finite element analysis code [12] indicates a directivity of 6.2 and $6.1 \mathrm{dBi}$ and radiating efficiency of -0.36 and $-0.31 \mathrm{~dB}$ at 870 and $915 \mathrm{MHz}$, respectively. The measured impedance indicates $\tau \approx 0 \mathrm{~dB}$ at $870 \mathrm{MHz}$ and $-0.4 \mathrm{~dB}$ at $915 \mathrm{MHz}$. Combining terms, we would expect a realized gain (the product of antenna gain and power transfer efficiency) of 5.9 and $5.4 \mathrm{dBi}$, respectively.

The free-space Friis equation can be modified for RFID transponders [13].

$$
r=\frac{\lambda}{4 \pi} \sqrt{\frac{P_{t} G_{t} G_{r} \tau \rho}{P_{\mathrm{th}}}}
$$

We following the convention of [13] that the subscript $t$ represents the transmitter (reader) and the subscript $r$ represents the receiver (tag); $\tau$ is the power transfer efficiency, $\rho$ is the polarization mismatch, and $P_{\text {th }}$ is the minimum (threshold) power to operate the IC. Commonly, readers use $6 \mathrm{dBi}$ circularly polarized antennas and tag antennas are linearly polarized, leading to a polarization loss of 0.5 . Using a published value of $P_{\mathrm{th}}=-13 \mathrm{dBm}$ [14], the maximum read distance of the tag from a commodity reader with $P_{t}=30 \mathrm{dBm}$ and $G_{r}=5.4 \mathrm{dBi}$ is approximately 9.7 meters (32 feet). Utilizing a $3 \mathrm{~dB}$ ground reflection, one could expect 13.7 meters (45 feet).

We performed an informal read distance experiment outdoors in an open field. The tag was first affixed to an expanded polystyrene sheet and held approximately one meter above the ground and far from any other object. We used a commodity reader at maximum FCC power settings (36 dBm EIRP) with a bistatic circularly-polarized antenna. The tag was moved away from the reader until we found the maximum distance at which the tag was detectable (maximum detectable distance). We anticipate a 3 $\mathrm{dB}$ increase in received power from a ground reflection. We found the maximum detectable distance to be 18.3 meters ( 60 feet). This exceeds our estimated maximum detection distance by $2.5 \mathrm{~dB}$. Next, we placed the antenna over a large ground plane (approximately 2 feet square) and observed the maximum detection increase to 20.5 meters (67 feet). The difference of about $1 \mathrm{~dB}$ change in directivity agrees with predictions based on simulation. Given those results, we would predict a free-space maximum detection distance of 12.9 and 14.5 meters (42 and 47 feet) without and with a large ground plane, respectively.

As a second method of validation, we placed the tag two meters from a different reader in a laboratory environment. The tag was placed on an expanded polystyrene block 2 meters from the reader. The reader frequency was fixed to $915 \mathrm{MHz}$, and the power was varied until we found the minimum power that was able to read the tag. Based on $-13 \mathrm{dBm}$ IC turn-on power, we would predict the minimum reader power would be $16.5 \mathrm{dBm}$. We measured $17.0 \mathrm{dBm}$. Given that result, we would predict a free-space read 


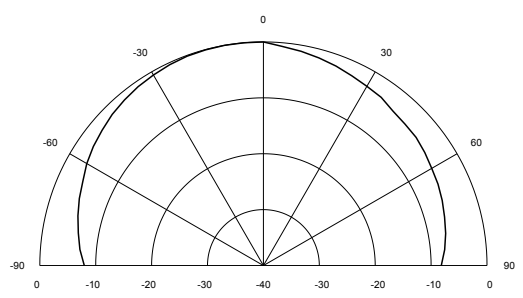

(a) E-plane, $870 \mathrm{MHz}$.

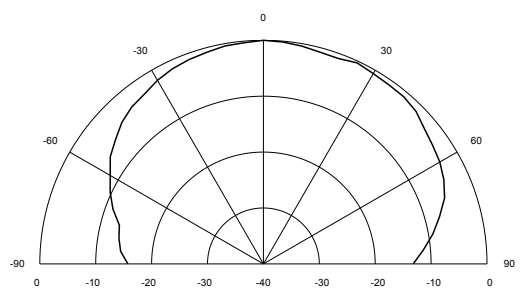

(c) E-plane, $915 \mathrm{MHz}$.

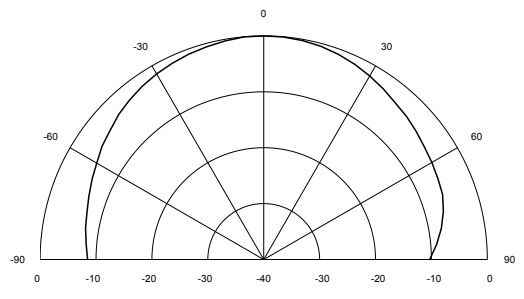

(b) H-plane, $870 \mathrm{MHz}$.

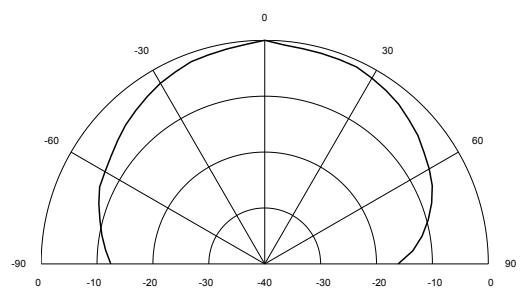

(d) H-plane, $915 \mathrm{MHz}$.

Fig. 4. Radiation pattern.

distance of 9.3 meters (32 feet). However, we have reason to believe that the system is not forward-link limited [4], and thus results in a conservative estimate. Regardless, we can conclude that the free-space read distance will be between 9.3 and 13.1 meters, and 10.4 and 14.7 meters on a large metal ground plane. Obviously, matched linearly polarized reader antennas will add $3 \mathrm{~dB}$ of link margin.

The measured radiation pattern of the tag in both the E- and H-plane at both 870 and $915 \mathrm{MHz}$ are shown in Fig. 4. Note that since the antenna is dual-polarized, the planes reverse at the two resonant frequencies. The radiation pattern was measured with the provided (not infinite) ground plane, and show good agreement with simulated results.

\section{CONCLUSION}

In this paper, we present a high-performance, dual-resonant, dual-polarized microstrip antenna. The antenna is a completely planar structure (avoids any cross-layer connections) so that the antenna may be easily manufactured using a traditional inlay. The antenna is designed to perform efficiently within the 865-868 and 902-928 MHz frequency bands, but we show how to modify the design to operate at any two of the three frequency bands used worldwide. We show measured impedance results using the power wave Smith chart, showing excellent power transfer efficiency across the frequency bands of interest. Measured results show an estimated free-space detection distance of between 9.3 and 13.1 meters. 


\section{ACKNOWLEDGMENT}

We are grateful for the helpful remarks from Kenneth R. Demarest and Daniel M. Dobkins.

\section{REFERENCES}

[1] D. M. Dobkins, The RF in RFID: Passive UHF RFID in Practice. Burlington, MA: Newnes, 2007.

[2] D. M. Dobkins and S. Weigand, "Environmental effects on RFID tag antennas," in IEEE MTT-S International Microwave Symposium, Long Beach, CA, Jun. 2005, pp. 4-7.

[3] J. Griffin, G. Durgin, A. Haldi, and B. Kippelin, "Radio link budgets for $915 \mathrm{MHz}$ RFID antennas placed on various objects," in Proc. 2005 Texas Wireless Symposium, Austin, TX, Oct. 2005, pp. 22-26.

[4] S. R. Aroor and D. D. Deavours, "Evaluation of the state of passive UHF RFID: An experimental approach," IEEE Systems Journal, vol. 1, no. 2, pp. 168-176, 2007.

[5] P. Bhartia, I. Bahl, R. Garg, and A. Ittipiboon, Microstrip Antenna Design Handbook. Artech House Publishers, Nov. 2000.

[6] H. Kwon and B. Lee, "Compact slotted planar inverted-F RFID tag mountable on metallic objects," Electronics Letters, vol. 41, no. 24, pp. 1308-1310, Nov. 2005.

[7] J. S. Chen and K.-L. Wong, "A single-layer dual-frequency rectangular microstrip patch antenna using a single probe feed," Microwave and Optical Technology Letters, vol. 11, pp. 83-84, Feb. 1996.

[8] K.-L. Wong and K.-P. Yang, "Small dual-frequency microstrip antenna with cross slot," Electronics Letters, vol. 33, no. 2, pp. 83-84, Nov. 1997.

[9] K. Kurokawa, "Power waves and the scattering matrix," IEEE Transactions on Microwave Theory and Technique, vol. 13, no. 3, pp. 194-202, Mar. 1965.

[10] P. V. Nikitin, K. V. S. Rao, S. F. Lam, V. Pillai, R. Martinez, and H. Heinrich, "Power reflection coefficient analysis for complex impedances in rfid tag design," IEEE Transactions on Microwave Theory and Technique, vol. 53, no. 9, pp. 2721-2725, Sep. 2005.

[11] M. Eunni, M. Sivakumar, and D. D. Deavours, “A novel planar microstrip antenna design for UHF RFID," Journal of Systemics, Cybernetics and Informatics, vol. 5, no. 1, pp. 6-10, Jan. 2007.

[12] Ansoft Corporation, HFSS Online Help, Ansoft Corporation, Pittsburg, PA, 2006.

[13] P. V. Nikitin and K. V. S. Rao, "Reply to 'Comments on "Antenna design for UHF RFID tags: A review and a practical application”,", IEEE Transactions on Antennas and Propagation, vol. 54, no. 6, pp. 1906-1908, Jun. 2006.

[14] T. Instruments, TI UHF Gen2 IC - Reference Guide, 1st ed., Dallas, TX, Jun. 2006. 